\title{
Aktuelle Trends und alternative Perspektiven der Hochschulentwicklung
}

Andreas Keller

\begin{abstract}
Unterfinanzierung der Hochschulen, Kommerzialisierung von Bildung und Forschung, Privatisierung der Studienfinanzierung, Flexibilisierung und Deregulierung der Beschäftigungsverhältnisse in der Wissenschaft und eine Studienstrukturreform nach „Schema F“, die weder den Anforderungen der Studierenden noch der beruflichen Praxis wirklich gerecht wird. Dies sind die wesentlichen Trends der hochschulpolitischen Entwicklung in Deutschland zu Beginn des 21. Jahrhunderts. Im Folgenden werden diesen Trends wissenschaftspolitische Alternativkonzepte und Gegenstrategien aus Sicht der Gewerkschaft Erziehung und Wissenschaft (GEW), der Bildungsgewerkschaft im DGB, gegenübergestellt.
\end{abstract}

\section{NACHFRAGE- UND BEDARFS- GERECHTER AUSBAU DER STUDIENPLÄTZE}

Statt heute ca. 1,9 Mio. könnten im Jahr 2020 rund 2,7 Mio. Menschen studieren folgt man den Prognosen der Kultusministerkonferenz. Auf die wachsende Nachfrage junger Menschen nach Studienplätzen hat die Politik aber noch keine überzeugende Antwort gefunden. Zwar haben sich die Regierungschefs von Bund und Ländern im Dezember 2006 auf Eckpunkte des „Hochschulpaktes 2020" verständigt. Sie scheinen jedoch - zum zweiten Mal nach dem „Öffnungsbeschluss“ von 1977 - der Illusion zu unterliegen, einen „Studentenberg untertunneln“ zu können. Mit dem Hochschulpakt sollen 90.000 zusätzliche Studienplätze finanziert werden. Fraglich ist nicht nur, ob hierfür die vorgesehenen ca. 140 Mio. $€$ pro Jahr ausreichen werden, sondern auch, wie eigentlich die restlichen rund 700.000 fehlenden Studienplätze geschaffen werden sollen.
Die im Herbst 2006 von Bundestag und Bundesrat verabschiedete Föderalismusreform verschärft zusätzlich das Problem der Unterfinanzierung der Hochschulen, statt es zu lösen. Im Rahmen der neuen Kompetenzverteilung zwischen Bund und Ländern hat sich der Bund aus seiner Verantwortung für die Entwicklung von Bildung und Wissenschaft gestohlen: Er zieht sich aus der Förderung des Hochschulbaus sowie aus erfolgreichen Bund-Länder-Programmen zurück. Insbesondere finanzschwache Länder werden auf Dauer nicht in der Lage sein, den Rückzug zu kompensieren. Die politisch gewollte Folge des Wettbewerbsföderalismus ist die Ausdifferenzierung der Hochschullandschaft in „Klasse“ und „Masse“. Die noch von Bundesbildungsministerin Edelgard Bulmahn (SPD) auf den Weg gebrachte „Exzellenzinitiative" begünstigt diese Entwicklung zusätzlich durch den gezielten Aufbau weniger Spitzenuniversitäten. Der Rückzug des Bundes aus der Hochschulfinanzierung in der Fläche und der gleichzeitige Einstieg in den Aufbau herausragender „Leuchttürme" sind somit zwei Seiten derselben Medaille.

Die internationalen Vergleichsstudien der OECD belegen: In Deutschland nehmen mit $35 \%$ eines Altersjahrgangs deutlich weniger junge Frauen und Männer ein Hochschulstudium auf als in anderen Industrieländern - neuerdings ist die Tendenz sogar wieder rückläufig. In Sonntagsreden ist es längst eine Binsenweisheit: Deutschland braucht in Zukunft nicht weniger, sondern deutlich mehr gut ausgebildete Akademikerinnen und Akademiker. Diesen guten Vorsätzen müssen jedoch Taten folgen. Eine elementare Voraussetzung für den überfälligen Anstieg der Studierenden- und Absolventenzahlen ist der nachfrage- und bedarfsgerechte Ausbau der Stu- dienplätze an den Hochschulen. Hierzu bedarf es einer gemeinsamen Kraftanstrengung von Bund und Ländern, die sich endlich auf eine nationale bildungspolitische Gesamtstrategie verständigen müssen. Gute Bildung und Chancengleichheit für alle - so lautet die bildungspolitische Leitlinie der GEW auch für die Hochschulen. Chancengleichheit hat dabei durchaus auch eine regionale Dimension: Der alte Verfassungsgrundsatz der Gleichwertigkeit der Lebensverhältnisse im Bundesgebiet muss auch in einer föderalisierten Hochschullandschaft zum Tragen kommen.

Die Voraussetzungen für einen bildungspolitischen Kurswechsel sind haushalts- und finanzpolitische Prioritätensetzungen für Zukunftsinvestitionen in Bildung und Forschung. Wie im LissabonProzess festgelegt, muss Deutschland endlich tatsächlich den Anteil seiner Bildungsausgaben am Bruttoinlandsprodukt von jetzt nur 2,5\% auf $3 \%$ steigern. Wenn die Politik schon nicht den Mut aufbringt, die Fehler der Föderalismusreform von 2006 zu korrigieren, sollte sie in der angekündigten Neuordnung der Finanzverfassung im Rahmen der bevorstehenden „Föderalismusreform II" wenigstens alle Länder befähigen, die ihnen vom Bund überlassenen bildungs- und wissenschaftspolitischen Aufgaben tatsächlich schultern zu können.

\footnotetext{
Andreas Keller, Dr., Leiter des Vorstandsbereichs Hochschule und Forschung beim Hauptvorstand der Gewerkschaft Erziehung und Wissenschaft (GEW). Arbeitsschwerpunkte: Hochschul- und Wissenschaftspolitik, Arbeitsplatz Hochschule und Forschung, Studienfinanzierung, Studienreform, Lehrerund Pädagogenausbildung. e-mail: andreas.keller@gew.de
} 


\section{AUTONOMIE OHNE AUTOKRATIE - INNOVATION DURCH PARTIZIPATION}

Der Mainstream der Bildungs- und Wissenschaftspolitik in Bund, Ländern und in supranationalen Organisationen steht heute für eine durchgreifende Kommerzialisierung von Bildung und Forschung. Bildung wird zunehmend nicht mehr als Menschenrecht, sondern als eine Ware begriffen, die auf einem im globalen Maßstab liberalisierten Markt gehandelt wird. Forschung, Lehre und Studium drohen auf eine abhängige Variable der ökonomischen Entwicklung reduziert zu werden. Die emanzipatorische Funktion von Bildung und die gesellschaftlichen Aufgaben von Forschung haben immer weniger Platz in den Hörsälen und Forschungslabors.

Hochschulen werden perspektivisch nicht mehr als Gemeinschaften von Lehrenden und Lernenden (Wilhelm von Humboldt) verstanden, sondern als Dienstleistungsunternehmen, die ihre Produkte Aus- und Weiterbildung sowie Forschungsdienstleistungen - auf dem Markt anbieten müssen. Wie Drittmittel- und Forschungsauftraggeber werden auch die Studierenden von Mitgliedern der Hochschulen zu deren Kunden und damit bereits begrifflich aus der Selbstverwaltungskörperschaft "Hochschule“ ausgegrenzt. Die in den 1960er und 1970er Jahren erkämpften Selbstverwaltungs- und Mitbestimmungsrechte von Beschäftigten, Studierenden, ja sogar Hochschullehrerinnen und Hochschullehrern werden abgebaut. Autokratische Leitungsorgane und externe Aufsichtsgremien ziehen nahezu alle Entscheidungsbefugnisse der auch in Haushaltsund Wirtschaftsfragen autonom gewordenen Hochschulen an sich.

Für die GEW bleibt es dabei: Bildung für jede Einzelne und jeden Einzelnen gehört zu den Grundvoraussetzungen einer entwickelten Gesellschaft. Sie darf nicht auf dem Markt gehandelt werden, sondern muss im Hinblick auf die gesellschaftlichen Aufgaben der Zukunft in politischer Verantwortung erhalten und ausgebaut werden. Bildung - auch Hochschulbildung - ist keine Ware, sondern ein Menschenrecht. Hochschulen müssen Selbstverwaltungskörperschaften in staatlicher Trägerschaft bleiben, die Forschung, Lehre und Studium auf Basis einer aufgabengerechten Arbeitsteilung autonom, aber in gesellschaftlicher Verantwortung organisieren.
Nach dem Motto „Autonomie ohne Autokratie“ ist die weitere Stärkung der Unabhängigkeit der Hochschulen zu unterstützen, wenn gleichermaßen die Partizipation der Hochschulmitglieder erweitert und die gesellschaftliche Verantwortung der Hochschulen institutionalisiert wird. Kuratorien und Hochschulräte dürfen nicht die ureigenen Selbstverwaltungsangelegenheiten der Hochschulen übernehmen. Sie müssen als eine Clearing-Stelle zwischen Hochschule und Gesellschaft agieren und die Wissenschaft mit den gesellschaftlichen Anforderungen an den Auftrag der Hochschulen in Forschung, Lehre, Studium und Weiterbildung konfrontieren. Ihnen sollten daher in erster Linie nicht unabhängige Sachverständige, sondern explizite Repräsentanten gesellschaftlicher Interessen - insbesondere auch der beruflichen Praxis einschließlich der Arbeitnehmerinnen und Arbeitnehmer angehören.

Nach dem Motto „Innovation durch Partizipation“ müssen die am Wissenschaftsprozess beteiligten Gruppen über wirksame Mitbestimmungsverfahren an der Steuerung der Hochschule beteiligt werden. Dies schließt keineswegs aus, dass die Partizipationsverfahren durch eine transparente Kompetenzverteilung zwischen gewählten Kollegialorganen (Entscheidung von Strukturen und Grundsätzen) und Exekutivorganen (Konkretisierung und Umsetzung der Grundsatzentscheidungen) effizienter und für die Hochschulmitglieder attraktiver gemacht werden. Hierzu können auch outputorientierte Steuerungsinstrumente (erfolgsorientierte Mittelverteilung auf Basis von Zielvereinbarungen) beitragen, wenn die Verfahren sowie Kriterien und Indikatoren der neuen Steuerungsmodelle selbst mitbestimmten Verfahren zugänglich sind. Auf allen Entscheidungsebenen muss Geschlechtergerechtigkeit als Hochschulaufgabe und Reformanliegen verinnerlicht werden und sich in verbindlichen Zielvorgaben und Maßnahmen niederschlagen.

\section{CHANCENGLEICHHEIT BEIM HOCHSCHULZUGANG UND IM STUDIUM}

Mit der Einführung von allgemeinen Studiengebühren ab dem ersten Semester haben die Regierungen von sieben Bundesländern - Baden-Württemberg, Bayern, Hamburg, Hessen, Niedersachsen, Nord-
rhein-Westfalen und das Saarland - bereits einen einschneidenden Schritt zur Privatisierung der Studienfinanzierung getan. Die schrittweise Annäherung an das Bezahlstudium in den letzten zehn Jahren durch Einführung von Einschreib- und Rückmeldegebühren, Langzeitstudiengebühren, Studienkonten oder Zweitstudiumsgebühren haben sich tatsächlich, wie von Anfang an befürchtet, als „Salamitaktik“ erwiesen, an deren Ende nun allgemeine Studiengebühren für alle stehen.

Parallel dazu vollzieht sich ein schleichender Funktionsverlust des Bundesausbildungsförderungsgesetzes (BAföG). Die Bundesregierung hat Anfang 2007 einen Entwurf für eine 22. BAföG-Novelle vorgelegt. Geplant sind lediglich systemimmanente Korrekturen an der Ausbildungsförderung, die für ausländische Studierende in Deutschland, für inländische Studierende im Ausland und für Studierende mit Kindern zwar Verbesserungen bringen sollen. Für Studierende, die über den zweiten Bildungsweg an die Hochschulen gelangt sind, sowie für zur Darlehensrückzahlung verpflichtete Eltern bedeuten sie jedoch Verschlechterungen. Die einst von der rotgrünen Bundesregierung in Aussicht gestellte Strukturreform ist ebenso wenig in Sicht, wie die längst überfällige, seit 2001 unterbliebene Anpassung der Fördersätze und Eltern-Freibeträge.

Bereits heute trägt das BAföG nur für eine Minderheit von $18 \%^{1}$ der Studierenden zur Finanzierung ihres Lebensunterhalts bei, und zwar mit durchschnittlich $375 €$ monatlich; kaum eine Studentin oder ein Student kann das Studium heute ausschließlich über das BAföG finanzieren. Obwohl in Deutschland ein zu geringer Anteil eines Altersjahrgangs ein Studium aufnimmt und abschließt, wird das Studium durch die Einführung von Studiengebühren und den sukzessiven Funktions-

\footnotetext{
Diese unschöne Quote versucht die Bundesregierung in ihren regelmäßig zu erstattenden BAföGBerichten seit 1982 dadurch zu verdecken, dass sie mit der sogenannten normativen Geförderten quote arbeitet, die die Zahl der BAföG-EmpfängerInnen nicht zur Zahl aller eingeschriebenen Studierenden, sondern zur Zahl der "dem Grunde nach BAföG-Berechtigten “ in Beziehung setzt. Da auf diese Weise eine Reihe von Studierenden aus der Statistik fällt (z. B. wenn sie das BAföGHöchstalter überschritten oder einen Fachrichtungswechsel ohne wichtigen Grund vollzogen haben), kann für 2005 eine Quote von 25,1 \% präsentiert werden.
} 
verlust des BAföG immer weiter verteuert. Die Chancengleichheit beim Hochschulzugang wird dadurch substanziell infrage gestellt. Insbesondere Kinder aus einkommensschwachen und bildungsfernen Familien werden vom Studium abgeschreckt: Der kapazitätsbedingte Numerus clausus, der sich aus der unzureichenden Hochschulfinanzierung ergibt, wird um einen sozialen Numerus clausus - als Folge einer unzureichenden Studienfinanzierung - erweitert. In kaum einem anderen europäischen Land ist der Zusammenhang zwischen sozialer Herkunft und Bildungsweg so ausgeprägt wie in Deutschland. Das zeigt auch die jüngste Sozialerhebung des Deutschen Studentenwerks: Von 100 Kindern aus der Herkunftsgruppe „niedrig“ nehmen nur elf ein Studium auf, bei der Herkunftsgruppe „hoch“ sind es 81.

Nicht ein Ausverkauf, sondern eine Erneuerung der Ausbildungsförderung ist daher überfällig. In den vergangenen 35 Jahren war das BAföG eine tragende und unersetzliche Säule der Studienfinanzierung. Dabei muss es bleiben. Als Erstes müssen die Fördersätze und Freibeträge regelmäßig angepasst werden, um den Empfängerkreis auszuweiten und die durchschnittliche Förderhöhe zu steigern. Zweitens muss die Ausbildungsförderung strukturell reformiert werden - mit dem Ziel einer bedarfsgerechten und elternunabhängigen Förderung ohne Rückzahlungsverpflichtung. Durch die Integration der Transferzahlungen des Familienlastenausgleichs, von denen heute nicht die Studierenden, sondern in größtem Maße Eltern mit hohen Einkommen profitieren, ließen sich die für die Strukturreform erforderlichen Mehraufwendungen zu einem relevanten Anteil gegenfinanzieren.

Verbesserungen in der Ausbildungsförderung dürfen aber nicht dadurch konterkariert werden, dass die Bundesländer Studiengebühren einführen. Die Bildungsgewerkschaft GEW bekräftigt ihre Ablehnung von Studiengebühren jeder Art und tritt weiterhin für die Gebührenfreiheit des Hochschulstudiums ohne Wenn und Aber ein. Sie unterstützt den studentischen Widerstand gegen die Einführung von Studiengebühren. Auch nach dem Urteil des Bundesverfassungsgerichts von 2005 und der Föderalismusreform gibt es keinen Automatismus für die Gebührenspirale - weder in rechtlicher noch in politischer Hinsicht. Den Ländern darf zwar - bis zu bestimmten Grenzen - von Bundesseite nicht mehr verwehrt werden, an ihren Hochschulen Gebühren zuzulassen. Aber kein Land wird zur Einführung von Gebühren gezwungen. Jede einzelne Landesregierung hat die Verantwortung dafür, dass aus dem leichtfertigen Experimentieren mit Studiengebühren kein nationaler Flächenbrand wird, der zu einer neuen deutschen „Bildungskatastrophe“ führt, und dass es stattdessen bei einem vorübergehenden Strohfeuer bleibt, das sich mittelfristig nicht gegen die bildungspolitische Vernunft im Interesse der Zukunftschancen der heranwachsenden Generation und der Zukunftsfähigkeit von Wissenschaft, Wirtschaft und Gesellschaft durchsetzen kann.

\section{WISSENSCHAFT ALS BERUF}

Die Arbeits- und Beschäftigungsbedingungen des Personals an den Hochschulen und Forschungseinrichtungen wurden seit den 1980er Jahren umfassend flexibilisiert und dereguliert. Unterhalb der Professur wird das wissenschaftliche Personal heute fast ausschließlich befristet beschäftigt; zunehmend werden Daueraufgaben in Lehre und Forschung auf nebenberuflich Tätige, wie Lehrbeauftragte oder wissenschaftliche Hilfskräfte, in prekären Beschäftigungsverhältnissen übertragen. Das neue Wissenschaftszeitvertragsgesetz der Großen Koalition weitet die befristeten Beschäftigungsverhältnisse nicht mehr nur des wissenschaftlichen, sondern zunehmend auch des nichtwissenschaftlichen Personals aus. Dadurch wird der Trend zur Umkehrung des im allgemeinen Arbeitsrecht geltenden Regel-Ausnahme-Verhältnisses zwischen unbefristet und befristet Beschäftigten weiter verstärkt. Die unmissverständliche Empfehlung der im März 2003 von der Europäischen Kommission beschlossenen Forschungs-Charta, ,dass die Leistung von Forschern nicht durch die Instabilität von Arbeitsverträgen beeinträchtigt" werden dürfe, wurde in diesem jüngsten Gesetzgebungsprojekt ganz offensichtlich ignoriert. Obwohl Föderalisierung und Deregulierung im Hochschulrecht sonst groß geschrieben werden, soll das Hochschulfristvertragsrecht die für 2008 vorgesehene Aufhebung des Hochschulrahmengesetzes durch Übertragung der Normen ins Zeitvertragsgesetz unbeschadet überstehen.

Die Personalstruktur ist insbesondere an den Universitäten durch übermäßig steile Hierarchien charakterisiert. Dies ist nicht aufgabengerecht und behindert die
Innovationsfähigkeit, Attraktivität und internationale Wettbewerbsfähigkeit der Hochschulen. Viele qualifizierte junge Menschen ziehen der wissenschaftlichen Laufbahn an einer deutschen Universität eine Karriere im Ausland oder in der Wirtschaft vor. Mit der Einführung von Juniorprofessuren als neuem Qualifikationsweg für die Hochschullehrerlaufbahn wurde zwar ein wichtiger Schritt gemacht, um die Hierarchien aufzubrechen und dem wissenschaftlichen Nachwuchs früher selbstständige Forschung und Lehre zu ermöglichen. Es wurde aber versäumt, den Juniorprofessorinnen und Juniorprofessoren Perspektiven für eine dauerhafte wissenschaftliche Arbeit zu geben. Vielen ist das Risiko, am Ende ihrer wissenschaftlichen Laufbahn in einer Sackgasse zu stecken, zu groß.

Dass in der Wissenschaft stabile und langfristige Berufsperspektiven fehlen, wirkt sich insbesondere für Frauen benachteiligend und abschreckend aus. Trotz leichter Fortschritte ist es immer noch nicht gelungen, die strukturellen Benachteiligungen von Frauen an den Hochschulen abzubauen. Nicht einmal jede zehnte Professur mit der höchsten Besoldungsgruppe C4 bzw. W3 ist mit einer Frau besetzt.

Wer die Qualität von Forschung und Lehre an den Hochschulen sichern und optimieren möchte, muss auch die Arbeitsbedingungen ihres Personals verbessern. Das ist der Ausgangspunkt für das alternative Reformkonzept der GEW. Wissenschaftlerinnen und Wissenschaftlern muss die Ausübung von Wissenschaft als Beruf eigenständig, selbstständig und auf Dauer möglich sein - auch außerhalb der professoralen Ebene. Der Arbeitsplatz „Hochschule und Forschung" muss attraktiver für qualifiziertes wissenschaftliches und nichtwissenschaftliches Personal werden. Wissenschaftlerinnen und Wissenschaftlern, die sich für eine Professur qualifiziert haben, muss eine echte berufliche Perspektive geboten werden, auch wenn sie nicht auf eine Professur berufen werden (in den USA ist dieses Prinzip als tenure track bekannt). Die Qualifizierung des wissenschaftlichen und künstlerischen Nachwuchses sollte in der Regel im Rahmen von Beschäftigungsverhältnissen erfolgen. Die Promotion ist kein verlängertes Studium und daher als erste Phase der wissenschaftlichen Berufsausübung anzuerkennen.

Die Voraussetzungen für die Befristung von Beschäftigungsverhältnissen im Hoch- 
schulbereich dürfen nicht länger einseitig vom Staat per Bundesgesetz oktroyiert werden. Sie müssen - wie in anderen Branchen üblich - von Arbeitgebern und Gewerkschaften in einem Tarifvertrag ausgehandelt werden. Die GEW fordert daher die Aufhebung der Tarifsperre im neuen Wissenschaftszeitvertragsgesetz, die abweichende tarifliche Regelungen untersagt. Damit gäbe es ausreichend Möglichkeiten, um den „Besonderen Teil Wissenschaft“ im Tarifvertrag des öffentlichen Dienstes für die Beschäftigten der Länder (TV-L) zu konkretisieren. Einer sachgerechten und wissenschaftsadäquaten Regelung der Arbeitsbeziehungen an den Hochschulen stünde nichts mehr im Wege.

\section{QUALITATIVE STUDIENREFORM IM EUROPÄISCHEN HOCHSCHULRAUM}

Trotz vieler positiver Zielsetzungen weist die Umsetzung des Bologna-Prozesses in Deutschland in vielen Studienfächern und an vielen Hochschulen eine zentrale Schwäche auf: Es droht eine Zweiteilung des Hochschulstudiums in ein Schmalspurstudium für die Masse und ein Exzellenzstudium für eine Elite. Hierfür sorgen formale und materielle Hürden, die den Übergang vom Bachelor- zum als Zweitstudium deklarierten Masterstudium erschweren. Nichtkonsekutive Masterstudiengänge - also Studiengänge, die nicht auf einem vorausgegangenen fachidentischen Bachelorstudiengang aufbauen erweisen sich auch in Ländern, die grundsätzlich an der Studiengebührenfreiheit festhalten, häufig als Einfallstor für Gebühren; analog gibt es auch einen Anspruch auf Förderung nach BAföG nur für konsekutive Studiengänge. Hinzu kommt, dass sich die Kultusministerkonferenz in ihren Strukturvorgaben für Bachelor- und Masterstudiengänge darauf verständigt hat, den Zugang zum Master ,von weiteren besonderen Zugangsvoraussetzungen " abhängig zu machen: Aufnahmeprüfungen, Bestnoten im Bachelor oder starre Quotenregelungen (z. B. $30 \%$ der Bachelorabsolventen) sind möglich.
Die Qualität der neuen Bachelorstudiengänge, die nach Maßgabe der BolognaErklärung berufsqualifizierend sein sollen, sowie ihre Akzeptanz auf dem Arbeitsmarkt sind nach wie vor unsicher. Angesichts dieses Umstandes ist es besonders fatal, dass nicht alle Studierenden nach Abschluss eines Bachelorstudiums ihr Studium mit dem Master fortsetzen können, wenn sie dies gerne möchten. Bund und Länder haben zwar bereits 1999 ein System zur Akkreditierung der neuen Studiengänge eingerichtet, noch trägt aber nur eine Minderheit tatsächlich ein Akkreditierungssiegel. Fragwürdig erscheint vor diesem Hintergrund die Entschlossenheit von Bund und Ländern, die Umstellung der Studienstrukturen - von wenigen Ausnahmen wie der Medizin oder der Rechtswissenschaft abgesehen - komplett und flächendeckend bis 2010 auf das BachelorMaster-Modell umzustellen. Obwohl die Forderung nach Wettbewerb in der Hochschulpolitik sonst hoch im Kurs steht, traut man den neuen Studienstrukturen nicht einmal für eine Übergangszeit den Wettbewerb mit den traditionellen einphasigen Studiengängen zu.

Die Alternative zur übereilten Anpassung der deutschen Studienstrukturen an ein schematisches Bachelor-Master-Modell wäre eine wirklich qualitative Studienreform im europäischen Hochschulraum. Im Sinne einer Entschleunigung des BolognaProzesses sollte dort, wo die Akzeptanz in den Fächern, bei den Studierenden und auf dem Arbeitsmarkt noch nicht gegeben ist, für eine über 2010 hinaus verlängerte Übergangszeit der plurale Wettbewerb alter und neuer Studienstrukturen zugelassen werden. Die Qualität und die Berufsbefähigung neuer wie alter Studiengänge müssen in transparenten Verfahren gesichert werden, an denen Lehrende und Lernende sowie Gewerkschaften und Arbeitgeber als Vertreter der beruflichen Praxis beteiligt sind. Der Übergang von Bachelor- zu Masterstudiengängen muss absolut durchlässig sein: Allen erfolgreichen Bachelorabsolventen, die weiter studieren möchten, muss ein Studienplatz in einem weiter- führenden Studiengang angeboten werden. Auch ein Wechsel der Hochschularten sollte bei diesem Übergang problemlos möglich sein: Der Bologna-Prozess könnte auf diese Weise zur Integration des tertiären Bildungssystems mit Universitäten und Fachhochschulen als unterschiedlichen, aber gleichwertigen Institutionen beitragen.

\section{VOM ABWEHRKAMPF ZUM GEGENENTWURF}

Die Gewerkschaften können die hochschul- und forschungspolitischen Herausforderungen am besten dann erfolgreich bewältigen, wenn sie nicht bei einem Abwehrkampf gegen die „Untertunnelung des Studentenberges“, gegen die Kommerzialisierung von Bildung und Forschung, gegen die Privatisierung der Studienfinanzierung, gegen die Flexibilisierung der Arbeits- und Beschäftigungsbedingungen oder gegen die Einführung von Schmalspurstudiengängen stehen bleiben, sondern einen zukunftsorientierten Gegenentwurf für eine alternative, demokratische Reform des Bildungs- und Wissenschaftssystems entwickeln. Die Bildungsgewerkschaft GEW hat nicht das Ziel, das Hochschulwesen, wie es Ende des 20. Jahrhunderts verfasst war, unverändert ins 21. Jahrhundert zu retten. Es geht vielmehr darum, eigene Vorstellungen für Veränderungen im Interesse der Arbeitnehmerinnen und Arbeitnehmer, der Lehrenden und Lernenden sowie der Gesellschaft umzusetzen. Diese möchte die GEW mit den anderen DGB-Gewerkschaften, mit den Wissenschaftsorganisationen, mit den Studierenden, Wissenschaftlerinnen und Wissenschaftlern diskutieren, weiterentwickeln und in den aktuellen Reformprozess einbringen. Ganz im Sinne des Mottos der jüngsten DGB-Kampagne: „Das geht besser. Aber nicht von allein!“ 